\title{
SUPLÊNCIA VASCULAR DO SEXTO ARCO COSTAL ATRAVÉS DOS MÚSCULOS PEITORAL MENOR E SERRÁTIL ANTERIOR
}

\author{
VASCULAR SUPPLY THROUGH AN OSTEOMUSCULAR FLAP OF THE MINOR \\ PECTORAL AND ANTERIOR SERRATUS MUSCLES
}

\author{
Alexandre Pesciotto ${ }^{1}$ \\ Carlos Augusto Gonçalves Pasqualucci ${ }^{3}$ \\ Carlos Alberto Marcovechio Fonseca ${ }^{1}$ \\ Josias de Andrade Sobrinho ${ }^{4}$ \\ Abrão Rapoport,TCBC-SP ${ }^{2}$
}

\begin{abstract}
RESUMO: Objetivo: Estudar a suplência vascular do sexto arco costal, através de injeção de corante no segmento da artéria axilar que nutre os músculos peitoral menor e serrátil anterior. Método: Realizada dissecção de retalho osteomuscular em 20 cadáveres frescos, 19 do sexo masculino e um do sexo feminino, com idade variando de 43 a 60 anos e peso entre 55 a $80 \mathrm{~kg}$, contendo os músculos peitoral menor na parte cranial e o serrátil anterior na parte caudal, além da sexta costela na parte distal. Com o retalho elevado e destacado do tórax, e as aderências osteomusculares intactas, foi injetado sob pressão um corante hidrossolúvel - látex azul - na artéria axilar até observar-se a coloração dos vasos periostais. $\mathrm{O}$ arco costal foi fotografado com os vasos periostais corados e descalcificado em solução de ácido nítrico a 5\% e diluído em formalina a 10\% para estudo histológico. Resultados: Em todos os cadáveres dissecados encontramos positividade pelo corante nos vasos periostais, como comprovação histológica. Conclusões: A presença de corante nos vasos periostais demonstra que esta rota de fluxo sangüínea pode viabilizar um retalho osteomuscular para reconstrução de face e mandíbula.
\end{abstract}

Descritores: Suplência vascular; Periostal; Arco costal; Peitoral menor; Serrátil anterior.

\section{INTRODUÇÃO}

As reconstruções após cirurgias para tratamento de tumores malignos iniciaram-se com Tansini no início do século $\mathrm{XX}^{1,2}$, que usou o músculo grande dorsal para recobrir as áreas de mastectomia e de linfadenectomia axilar. Este cirurgião estabeleceu um dos princípios da cirurgia oncológica, que é a retirada do tumor com margem de segurança através da remoção dos tecidos locais e também dos linfonodos regionais para tratamento e prevenção das recidivas locais e da disseminação metastática. As reconstruções são importantes para a reabilitação precoce dos pacientes, pois as cirurgias para tratamento dos tumores são, em geral, mutilantes. Há necessidade de tecidos viáveis e livres de neoplasia. A artéria axilar forne- ce uma grande fonte de tecidos ósseos, musculares e epidérmicos para reconstruções.

As reconstruções de cirurgias de cabeça e pescoço por intermédio de retalhos miocutâneos, em um único estágio, podem ser realizadas com o músculo peitoral maior para reparações complexas das regiões cervicais e intraorais ${ }^{3}$. Todavia, há necessidade de métodos alternativos no arsenal da cirurgia de cabeça e pescoço. Outros músculos foram descritos e também irrigados através da artéria axilar. O músculo peitoral menor, pediculado, pode ser empregado para reconstrução em cabeça e pescoço com o sexto arco costal ${ }^{4,5}$. O músculo serrátil anterior tem algumas peculiaridades que o torna versátil em cirurgias reconstrutivas e pode ser empregado isoladamente, como um retalho pediculado englobando todas as suas digita-

1. Cirurgião do Hospital Heliópolis, São Paulo

2. Coordenador do Curso de Pós-graduação do Hospital Heliópolis, São Paulo

3. Doutor em Patologia (FMUSP) e Diretor do Serviço de Verificação de Óbitos (SVO) da Cidade de São Paulo

4. Cirurgião do Hospital Heliópolis, São Paulo.

Recebido em 20/07/2001

Aceito para publicação em 05/03/2002

Trabalho realizado no Serviço de Verificação de Óbitos (SVO) da Cidade de São Paulo. 
ções ${ }^{6}$, ou somente as digitações inferiores ${ }^{7}$. Pode ser empregado como um retalho livre com as digitações inferiores ${ }^{8,9} \mathrm{e}$ também associado ao músculo grande dorsal ${ }^{10}$. Portanto, o uso deste músculo é recente e deveria ter uma maior divulgação $0^{6,10-12}$

A artéria axilar é fonte de vários retalhos ósseos vascularizados, como a margem lateral da escápula, através da artéria circunflexa da escápula, os arcos costais posteriores através do músculo grande dorsal, os arcos costais da região torácica anterior através dos músculos peitoral maior, menor e serrátil anterior com suas digitações inferiores e também do ângulo inferior da escápula, pela inserção do músculo serrátil anterior neste local.

A reconstrução mandibular tem no retalho osteomuscular uma grande utilidade, principalmente com a costela e seu pedículos, entre eles o retalho composto pelo músculo serrátil anterior que, gradualmente, tem sido incorporado ao arsenal cirúrgico ${ }^{13-15}$. Este retalho apresenta um longo pedículo através dos ramos da artéria toracodorsal e situa-se na parte anterior do tórax, facilitando o ato operatório, pois o paciente permanece em uma única posição durante todo o ato cirúrgico. Pode ser realizado pela mesma equipe da intervenção oncológica, no caso de reconstrução com o músculo serrátil anterior, assim como com os músculos peitoral maior, menor e grande dorsal.

Os ossos costais têm o suprimento sangüíneo de várias fontes ${ }^{16-19}$. A artéria nutriente principal é ramo inicial da artéria intercostal posterior, ramo da aorta torácica, que penetra na cabeça da costela, percorre e irriga a medula óssea e os dois-terços internos do tecido ósseo cortical - chamada de nutrição endostal. Um segundo sistema, menos importante, é derivado das artérias intercostais (anteriores e posteriores) que nutrem, principalmente, o tecido periostal e parte da cortical óssea adjacente - chamada de nutrição intercostal/periostal. Este periósteo também tem parte da sua nutrição através dos tecidos musculares que originam-se no osso $\operatorname{costal}^{18}$ - denominada nutrição supracostal. Os vasos periostais penetram na substância cortical através de pequenos forames ${ }^{19} \mathrm{e}$, nas camadas internas, as ramificações comunicam-se com os vasos da medula óssea formando conexões anastomóticas. Deste modo, os vasos periostais são capazes de irrigar toda a substância cortical óssea, como nos casos de retalhos osteomusculares.

Nosso estudo é uma adaptação ao uso de retalho muscular, contendo na parte superior o músculo peitoral menor $^{4,5}$ e na parte inferior o músculo serrátil anterior com suas digitações inferiores ${ }^{7}$ e o sexto arco costal na parte distal, para avaliar o fluxo arterial na circulação periostal.

\section{MÉTODO}

Este estudo foi realizado no Serviço de Verificação de Óbitos da Cidade de São Paulo. Ofício Número 55/99. Foram estudados 20 cadáveres frescos, até 12 horas após o óbito, sendo um do sexo feminino e 19 do sexo masculino. A idade variou de 43 a 60 anos, o peso de $55 \mathrm{~kg}$ a $80 \mathrm{~kg}$, a altura variou de $1,60 \mathrm{~m}$ a $1,90 \mathrm{~m}$.
O lado direito foi dissecado em 19 cadáveres e o lado esquerdo, em um cadáver.

O músculo peitoral menor situa-se na parte cranial do retalho; o músculo serrátil anterior, com suas digitações inferiores, na parte caudal, e o sexto arco costal localiza-se na parte distal do retalho (Figura 1).

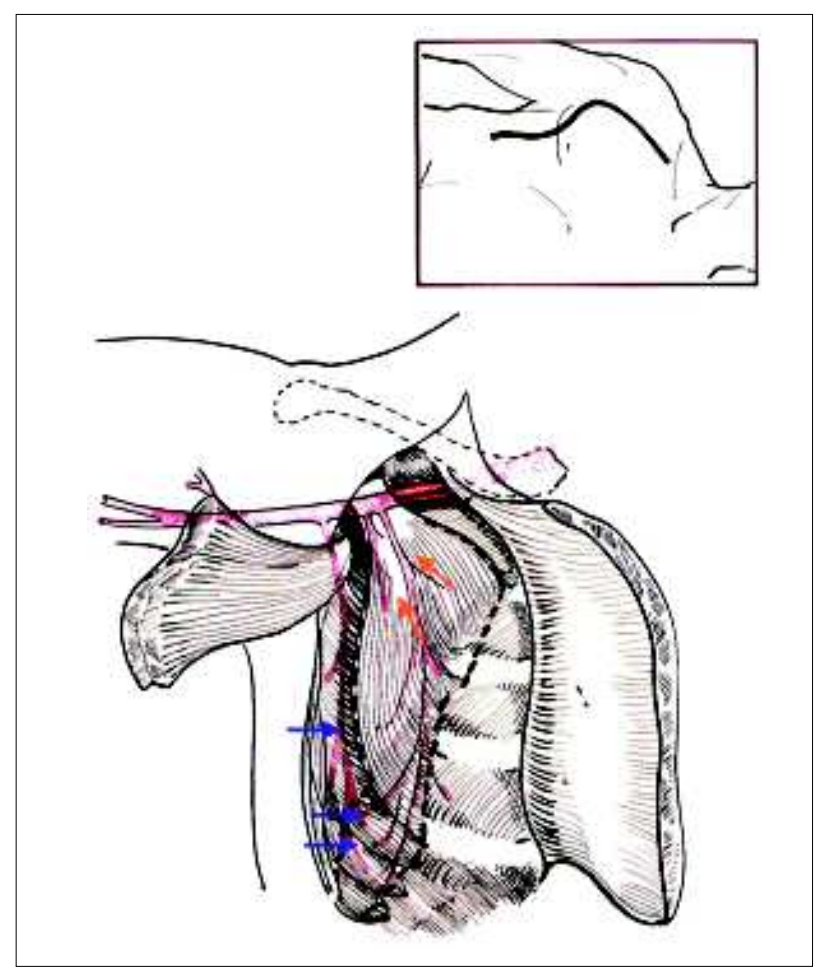

Figura 1 - Ilustra a artéria axilar em vermelho e seus ramos para o músculo peitoral menor (setas laranjas) através das artérias torácica lateral e os ramos peitorais posteriores na parte medial. A artéria toracodorsal (setas azuis), com os ramos para o músculo serrátil anterior situa-se na parte lateral. A linha pontilhada demarca o músculo peitoral menor na parte superior e as digitações inferiores do músculo serrátil anterior na parte inferior. (Ilustração do Dr. Edgard Bolanho.)

O cadáver foi mantido em decúbito dorsal com o braço em abdução de $90^{\circ}$. Uma incisão arciforme da pele foi feita iniciando-se na segunda articulação costocondral, até a região axilar anterior, continuando-se em direção à crista ilíaca até alcançar o hipocôndrio (Figura 1, no alto à direita). A dissecção inicial foi feita sobre as fáscias dos músculos peitoral maior, oblíquo externo do abdome, grande dorsal e serrátil anterior, expondo todo o hemitórax anterior. A inserção do músculo peitoral menor no processo coracóide da escápula foi desinserida, expondo o feixe vasculonervoso da região axilar. A artéria axilar foi dissecada em toda sua extensão e ligada na emergência do tórax com fio de algodão, preservando o ramo toracoacromial.

$\mathrm{O}$ feixe vasculonervoso da região axilar foi dissecado até o tendão do músculo grande dorsal. Após a emergência do tronco subescapular da artéria axilar, foi intro- 
duzida uma sonda de material plástico número 14 no interior da artéria axilar, que também foi ligada neste nível com fio de algodão, juntamente com o cateter (Figura 2). O segmento arterial incluiu a artéria toracoacromial na parte medial e a artéria subescapular na parte lateral. As três partes da artéria axilar estavam neste segmento vascular e o cateter estava posicionado no seu interior, com as extremidades medial e lateral ligadas para direcionar o corante somente aos ramos arteriais compreendidos neste segmento arterial.

A artéria subescapular e seu ramo toracodorsal esta-

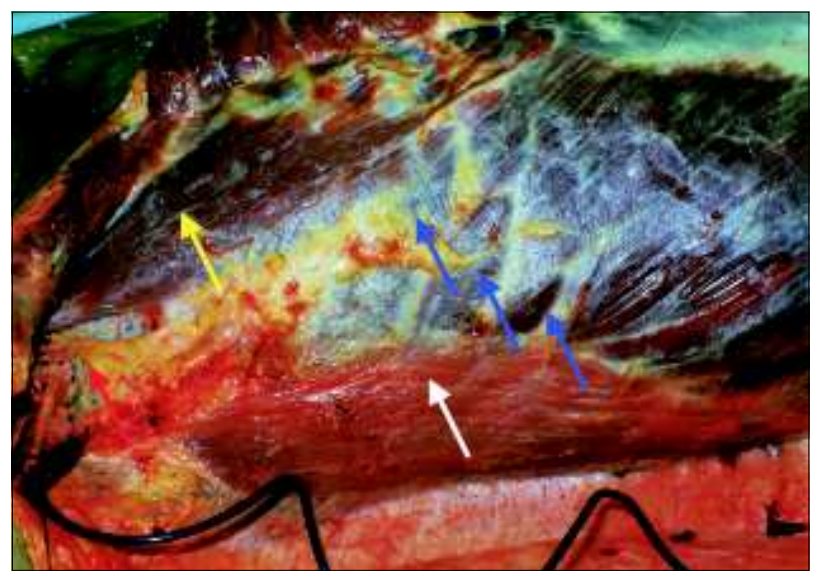

Figura 2 - Fotografia de cadáver expondo a parede medial do tórax. Músculo peitoral maior seccionado (seta preta). Músculo peitoral menor na parte superior (seta amarela), $m$. grande dorsal na parte inferior (seta branca) e $m$. serrátil anterior entre eles (setas azuis). Artéria axilar contendo cateter número 14 no seu interior e ligada nas partes medial e lateral (seta vermelha).

vam incluídas nestes tecidos, além dos ramos para o músculo peitoral menor. A artéria circunflexa da escápula foi ligada e o ramo para o músculo grande dorsal da artéria toracodorsal também foi ligado.

Os ramos arteriais originados da artéria toracodorsal para o músculo serrátil anterior foram preservados nesta parte inferior do retalho. Com a artéria toracodorsal incluída no retalho, as digitações inferiores do músculo serrátil anterior foram dissecadas a partir da quarta digitação. As digitações inferiores (quinta, sexta, sétima e oitava) foram anexadas ao retalho após serem desinseridas do ângulo inferior da escápula e dos ossos costais, exceto a origem da sexta digitação do músculo serrátil anterior no sexto arco costal.

O retalho foi conectado somente ao sexto arco costal e aos músculos intercostais anteriores acima e abaixo desta costela para manutenção da continuidade anatômica e vascular. Com o retalho destacado do tórax e mantido pelo segmento da artéria axilar com seus ramos para os músculos peitoral menor e serrátil anterior, injetamos inicialmente $20 \mathrm{ml}$ de água oxigenada para dissolver coágulos na circulação arterial e dilatar os vasos distais e, em seguida, foi injetado o corante - látex - sob pressão manual, com uma seringa de $50 \mathrm{cc}$. O cateter número 14 localiza-se no interior das três partes da artéria axilar. O corante foi injetado até o periósteo iniciar a coloração, que foi observada na face interna do osso costal e também nos músculos intercostais anteriores. Houve grande extravasamento do corante na face externa e na face interna dos músculos peitoral menor e serrátil anterior, e estes vasos foram ligados individualmente para evitar uma rota alternativa do fluxo arterial. Em alguns casos, todo o retalho foi retirado do cadáver para dissecção da artéria axilar e seus ramos para os músculos peitoral menor e serrátil anterior (Figura 3).

Ao final dos procedimentos de dissecção realizamos

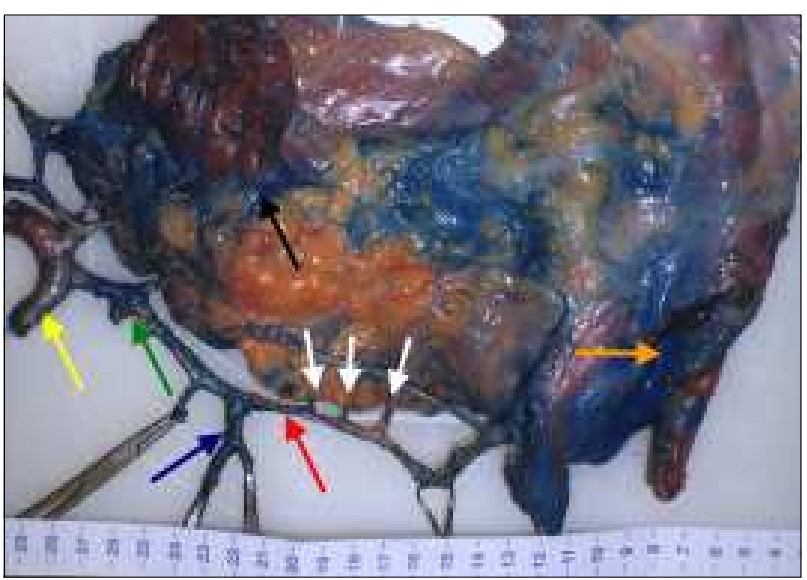

Figura 3 - Produto da dissecção da peça anatômica (face ventral), contendo: 1 - artéria axilar (seta amarela) com seus ramos para $m$. peitoral menor, 2 - tronco subescapular (seta verde) com os ramos circunflexos da escápula (seta azul) e a. toracodorsal (seta vermelha), 3 - ramos para o m. serrátil anterior (setas brancas), 4-m. peitoral menor (seta preta), 5 - m. serrátil anterior e o sexto arco costal (seta laranja).

estudo histológico do sexto arco costal, após descalcificação e coloração pela hematoxilina e eosina (HE).

\section{RESULTADOS}

Em todos os casos dissecados (20 cadáveres) observou-se corante nas artérias periostais da sexta costela (Figura 4).

Através da microscopia óptica confirmamos a presença do corante (látex azul) no lúmen das artérias periostais do sexto arco costal (Figura 5), documentando com a realização de fotomicrografia.

\section{DISCUSSÃO}

Os cirurgiões que tratam tumores de cabeça e pescoço não devem comprometer a extensão da ressecção para favorecer a reconstrução imediata ou tardia dos pacientes. A extirpação do tumor, com margens de segurança, deve ser o objetivo principal e, após o ato operatório, as várias 


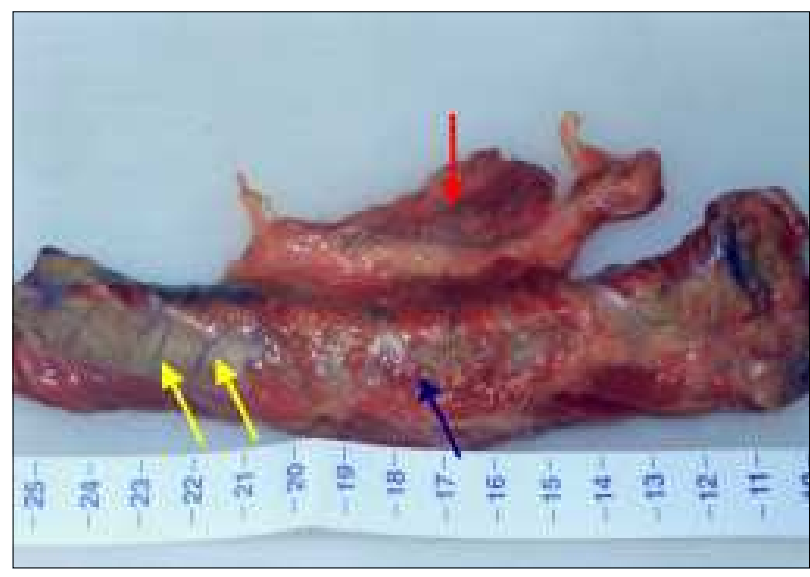

Figura 4 - Fotografia do sexto arco costal com corante - látex azul - nos vasos periostais (setas amarelas). Inserção do músculo serrátil anterior no arco costal (seta azul) e $m$. serrátil anterior (seta vermelha).

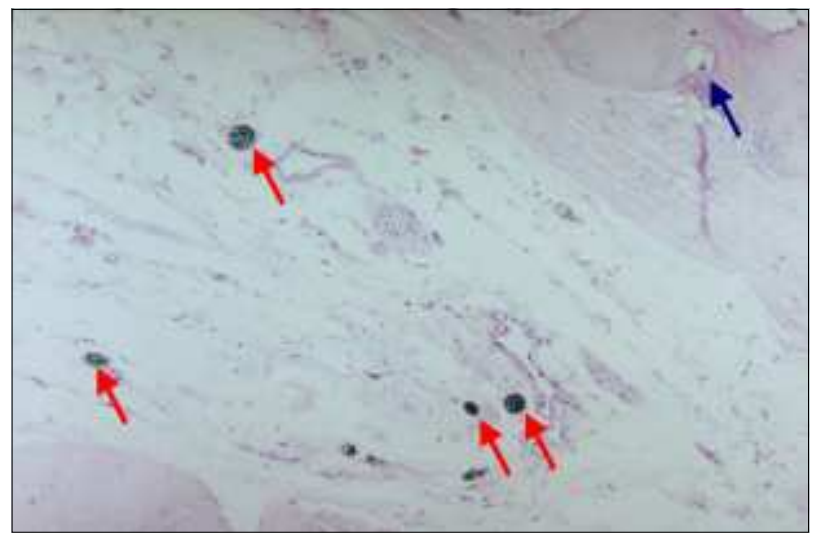

Figura 5 - Fotomicrografia de corte histológico de tecido periostal do sexto arco costal apresentando corante - látex azul - no interior de artérias periostais (setas vermelhas). Tecido ósseo descalcificado e corado (HE) 100XAO (seta azul).

opções de reconstrução devem ser consideradas. As reparações dos tecidos e da parte funcionais são importantes, assim como a aparência do paciente operado.

A perda da mandíbula causa deformidade permanente e estigmatizante, com danos irreversíveis que atingem todo o ambiente familiar e social. As reconstruções mandibulares são atenuantes do problema. Existem várias técnicas para tal: a) enxertos ósseos autógenos não vascularizados (cortical ou trabecular); b) materiais aloplásticos, como fios de Kirschner, próteses de titânio e de Dacron ${ }^{\circledR}$; c) aloenxertos, como osso irradiado; e d) retalhos ósseos vascularizados através de retalhos pediculados ou de retalhos livres por métodos microcirúrgicos. Destes, os melhores resultados são obtidos com o recurso da microcirurgia, porém requerem equipes altamente especializadas e demandam horas de cirurgia. As áreas ósseas doadoras mais comuns, além da fíbula ${ }^{20}$, são: 1 - clavícula; 2 - costela; 3 -ilíaco; 4 - rádio; 5 - escápula; 6- segundo metatarso. Os retalhos pediculados podem ser feitos pela mesma equipe da cirurgia dos tumores de cabeça e pescoço, uma vez que os especialistas em anastomoses vasculares são raros.

O músculo peitoral maior é consagrado como vetor de retalho ${ }^{3}$. Os músculos peitoral maior, menor, grande dorsal e serrátil anterior devem atualmente fazer parte do arsenal cirúrgico das equipes de cirurgias de cabeça e pescoço.

As variações anatômicas dos ramos da artéria axilar são freqüentes. Como nosso trabalho inclui a parte arterial situada entre as artérias toracoacromial e subescapular e incorpora os ramos destas artérias, quaisquer que forem as variações, todas elas serão englobadas pelo retalho proposto. A anatomia vascular desta região está bem documentada na literatura ${ }^{4,11,21,22}$.

$\mathrm{O}$ tronco arterial subescapular fornece uma grande variedade de vasos para reconstruções, que podem ser pediculadas ou através da microcirurgia, além de fornecer múltiplas subunidades segmentares em um único pedícu$1 \mathrm{o}^{23}$. O tronco subescapular nutre as digitações inferiores do músculo serrátil anterior, o músculo grande dorsal e também os territórios fasciocutâneos escapular e para-escapular, além da margem lateral da escápula. A artéria circunflexa da escápula permite que dois retalhos fasciocutâneos independentes sejam feitos na região dorsal. A artéria toracodorsal com o seu ramo para o músculo grande dorsal também permite a realização de dois retalhos musculares independentes ${ }^{23}$.

A irrigação para o músculo serrátil anterior fornece outra fonte de retalhos, portanto, o tronco arterial subescapular permite que todos os seus ramos possam ser fontes de retalhos e cada subunidade pode ser empregada de acordo com a necessidade da reparação. Estas subunidades têm irrigações distintas que favorecem reconstruções tridimensionais na face ${ }^{8,10-14,23}$. O ramo (ou ramos) para o músculo serrátil anterior nutre-o em sua parte inferior de um modo altamente segmentar e cada ramo emitido para suas digitações mantém conexões com o periósteo da costela na qual o músculo originou-se. Os vasos torácicos laterais são responsáveis pela nutrição das digitações superiores e também apresentam essas conexões músculo-periostais. Esta vascularização permite que as costelas sejam utilizadas como retalhos compostos osteomusculares.

A artéria subescapular apresenta variações quanto à sua origem na artéria axilar, que pode ser na terceira parte (a mais frequiente), na segunda parte e até na primeira parte $^{21,22}$. A artéria toracodorsal, ramo da artéria subescapular em $94 \%$ das vezes, pode originar-se diretamente da artéria axilar e até da artéria torácica lateral ${ }^{21,22}$. Como esta artéria é responsável pela nutrição da parte inferior do músculo serrátil anterior, estas variações anatômicas podem comprometer um retalho livre ou pediculado deste músculo. A artéria para o músculo serrátil anterior pode originar-se diretamente da artéria axilar e não da artéria toracodorsal $^{22}$. Apesar desta distribuição ser rara - somente um por cento das vezes -, se houver um planejamento ci- 
rúrgico com o músculo serrátil anterior com base na artéria toracodorsal, este procedimento será inviável. O retalho proposto através deste estudo, com os músculos peitoral menor e serrátil anterior, engloba todas as eventuais variações anatômicas na nutrição de ambos os músculos. Existem anastomoses entre a artéria toracodorsal com as artérias intercostais anteriores ${ }^{4,5,9,14,17,19,24}$. Nosso estudo demonstrou essa intercomunicação, com preenchimento de vaso intercostal anterior.

A superioridade dos retalhos ósseos vascularizados para reconstrução em cirurgias de cabeça e pescoço está bem documentada ${ }^{20}$. Os tecidos ósseos próximos à região cefálica são locais em que retalhos pediculados podem ser baseados nas artérias subclávia, axilar e cervicais. Como as costelas têm o suprimento arterial de várias fontes (endostal, intercostal/periostal e supracostal), estas vias geraram diferentes tipos de retalhos vascularizados e também uma controvérsia considerável sobre qual destas vias de nutrição seria capaz de suprir todo o tecido ósseo, no caso de intervenções com retalhos livres ou pediculados.

Durante o ato cirúrgico nos quais foram realizados retalhos ósseos vascularizados com base na circulação periostal, vários autores notaram que houve sangramento arterial proveniente dos vasos endostais das costelas seccionadas $^{4,5,10,14}$. Estas observações indicam a viabilidade da costela transplantada e que existem conexões vasculares do periósteo com o endósteo. Nosso estudo mostra, em todos os casos, vasos periostais corados através de corante injetado nas artérias nutrientes e estes vasos são o final da rota de fluxo através dos músculos peitoral menor e serrátil anterior.

Com a rica rede periostal demonstrada neste trabalho, podemos inferir que a microcirculação do periósteo será suficiente para a nutrição de todo o tecido ósseo, inclusive da medula óssea. O uso do músculo serrátil anterior torna-se grande opção para reconstrução osteomuscular. A vascularização para a costela com o músculo serrátil anterior está sempre presente, porém há alguma incerteza quando são usados os músculos peitoral maior ou grande dorsal $^{12}$.

O músculo peitoral menor foi estudado minuciosa- mente ${ }^{4,5}$ para a região da cabeça e pescoço. Seu uso como retalho osteomuscular é utilizado somente por alguns centros de cirurgias de cabeça e pescoço. As vantagens são: este retalho pode ser realizado pela mesma equipe cirúrgica, não há necessidade de mudança postural do paciente e os outros retalhos que a região axilar proporciona podem ser utilizados conjuntamente.

A superioridade dos retalhos ósseos vascularizados ${ }^{20}$ está documentada, inclusive com o uso de implantes dentários no mesmo ato operatório para reparação de pacientes portadores de tumores de cabeça e pescoço. A qualidade de vida dos pacientes deve estar presente nas decisões médicas, particularmente quando se trata de pacientes oncológicos, cujos tratamentos são agressivos e que a sobrevida permanece curta. Os retalhos pediculados osteomusculares vascularizados para reconstruções em cabeça e pescoço têm vantagens aparentes, pois podem reparar grandes defeitos em um único procedimento cirúrgico, porém há necessidade de se considerar que aumenta o tempo cirúrgico, que há uma morbidade na área doadora e que os vários retalhos descritos são, em geral, muito grandes e pesados. Há cirurgiões relutantes em aceitar, por outro lado, os retalhos livres com tecido ósseo vascularizado, porque estes, apesar de serem realizados no mesmo ato operatório, requerem uma outra equipe treinada em microcirurgia, prolongam o tempo cirúrgico e ainda existem índices de falhas neste método. Os retalhos ósseos vascularizados podem receber irradiação nas primeiras semanas após o ato operatório. Os retalhos musculares da parede torácica anterior são tecnicamente mais simples de serem executados e a vascularização óssea é mais segura.

Deste nosso estudo podemos inferir que o retalho osteomuscular da sexta costela, incluindo os músculos serrátil anterior e peitoral menor, é capaz de irrigar, com segurança, o periósteo do sexto arco costal a partir das conexões musculares, quando esta rota de fluxo arterial for a única via de irrigação sangüínea e que retalho osteomuscular pode ter aplicabilidade clínica como mais um recurso para reconstruções extensas de mandíbula e da parede torácica.

\section{REFERÊNCIAS}

\begin{abstract}
Background: Injecting some dye into the segment of the axilar artery which nourishes the minor pectoral and anterior serratus muscles we could evaluate the periostal circulation at the sixth costal arc. Method: We studied an osteomuscular flap in 20 fresh corpses, 19 of them males, with ages varying from 43 to 60 and weight between 55 and $80 \mathrm{Kg}$, with the minor pectoral muscle in the cranial portion and the anterior serratus in the caudal portion. With intact muscular adhesions, a water-soluble dye - blue latex - was injected into the axilar artery under pression until periostal vessel coloration could be seen. The costal arc was photographed with the dyed periosteous vessels and was decalcified in 5\% nitric acid solution thinned in $10 \%$ formalin for histological study of the periosteous. Results: In all of the dissected corpses positivity by the dye in periosteous vessels could be found and histologically proved. Conclusion: Blood fluid course throuh periosteous vessels can accomplish an osteomuscular flap on extensive reconstructions of face and mandible.
\end{abstract}

Key Words: Vascular supply; Periosteous; Costal arc; Minor pectoral; Anterior serratus. 
1. Tansini I. Sopra il mio nuovo processo di amputazione della mamella. Gaz Med Ital 1906; 57: 141

2. Tansini I. Nuovo processo per l'amputazione della mamella per cancre. Riforma Medica 1896; 12: 3.

3. Cuono CB, Aryian S. Immediate reconstruction of a composite mandibular defect with a regional osteomusculocutaneous flap. Plast Reconstr Surg 1980; 65: 477483.

4. Azevedo JF. Retalho osteomuscular de pequeno peitoral e costela para reconstrução do arco mandibular: uma técnica original. Rev Bras Cir Cab Pesc 1984; 8: 84 100 .

5. Azevedo JF. Pectoralis minor flap: an experimental study and clinical aplications of osteomuscular, osteomyocutaneous and myocutaneous models. Head \& Neck Surg 1987; 9: 211-222.

6. Arnold PG, Pairolero PC, Waldorf JC. The serratus anterior muscle : Intrathoracic and extrathoracic utilization. Plast Reconstr Surg 1984; 73: 240-248.

7. Inoue $\mathrm{T}$, Ueda $\mathrm{K}$, Hatoko A, Harashima $\mathrm{M}$. The pedicled extended serratus anterior myocutaneous flap for head and neck reconstruction. Br J Plast Surg 1991; 44: 259-265.

8. Takayanagi S, Tsukie T. Free serratus anterior muscle and myocutaneousflaps. Ann Plast Surg 1982; 80: 277283.

9. Richards MA, Poole MD, Godfrey AM. The serratus anterior/rib composite flap in mandibular reconstruction. Br J Plast Surg 1985; 38: 466-477.

10. Harii K, Yamada A, Ishihara K, Miki Y, Itoh M. A free transfer of both latissimus dorsi and serratus anterior flaps with thoracodorsal vessel anastomoses. Plast Reconstr Surg. 1982 ; 70: 620-629.

11. Cuadros CL, Driscol CLW, Rothkopf DM. The anatomy of the lower serratus anterior muscle: A fresch cadaver study. Plast Reconstr Surg 1995; 95: 93-97.

12. Netscher D, Alfort EL, Wigoda P, Cohen V. Free composite myo-osseous flap with serratus anterior and rib: Indications in head and neck reconstruction. Head \& Neck 1998; 20: 106-112.

13. Whitney TM, Buncke HJ, Alpert BS, Buncke GM, Lineaweaver WC. The serratus anterior free-muscle flap: Experience with 100 consecutive cases. Plast Reconstr Surg 1990; 86: 481-490.

14. Bruck JC, Bier J, Kistler D. The serratus anterior osteocutaneous free flap. J Reconstr Microsurg 1990; 6: 209 213.

15. Fassio E, Mortillet S, Laulan J, Laurent B, Fouquet B, Goga D, Ballon G. Lambeau de muscle serratus anterior: Indications et séquelles. A propos de 26 cas et revue de la littérature. Ann Chir Plast Esthet 1999; 44: 175182.

16. Ariyan S, Finseth FJ. The anterior chest approach for obtai- ning free osteocutaneous rib flap. Plast Reconstr Surg 1978; 62: 676-685.

17. Ariyan $\mathrm{S}$. The viability of rib grafts transplanted with periostal blood suplly. Plast Reconstr Surg 1980; 65: 140 -151 .

18. Hendel PM, Hattner MD, Rodrigo J, Buncke HJ. The funcional vascular anatomy of rib. Plast Reconstr Surg 1982; 70: 578-585.

19. Hui KCW, Zhang F, Lineaweaver WC, Moon W, Buncke GM, Buncke HJ. Serratus anterior-rib composite flap: Anatomic studies and clinical application to hand reconstruction. Ann Plast Surg 1999; 42: 132-136.

20. Foster RD, Anthony JP, Sharma A, Pogrel MA. Vascularized bone flaps versus nonvascularized bone grafts for mandibular reconstruction: An outcome analysis of primary bone union and endosseous implant success. Head \& Neck 1999; 21: 66-71.

21. Bartlett SP, May JW, Yaremchuk MJ. The latissimus dorsi muscle: A fresh cadaver study of the primary neurovascular pedicle. Plast Reconstr Surg 1981; 67: 631636.

22. Rowsell AR, Davies DM, Eisenberg N, Taylor GI. The anatomy of the subscapular-thoraco-dorsal arterial system: study of 100 cadaver dissections. Br J Plast Surg 1984; 37: 574-576.

23. Tobin GR, Moberg A, Ringberg A, Netscher D. Mandibular-facial reconstruction with segmentally split serratus anterior composite flaps. Clin Plast Surg 1990; 17: 663-672.

24. Salles FA, Ribak S, Dalapria R, Resende MR, Andrade D, Toledo CS. Estudo anátomo-radiológico do retalho do músculo serrátil anterior. Rev Bras Ortop 1994; 29: 361-364.

Endereço para correspondência:

Prof. Dr. Abrão Rapoport

Praça Amadeu Amaral, 47 - cj. 82

01327-010 - São Paulo-SP

E-mail: cpgcp.hosphel@attglobal.net 